\title{
A QUEUEING BASED TRAFFIC FLOW MODEL
}

\author{
Nico Vandaele*, T om Van Woensel* and Aviel Verbruggen*** \\ *UFSIA, Prinsstraat 13, 2000 Antwerp, Belgium \\ *UFSIA, Prinsstraat 13, B2000 Antwerp, Belgium \\ **UFSIA-STEM, Kleine K auwenberg 12, 2000 Antwerp, Belgium \\ Corresponding Author: \\ Tom Van Woensel, \\ Prinsstraat 13, B-2000 Antwerp, Belgium, +32 32204069 \\ Tom.VanWoensel@ufsia.ac.be
}

Published in:

Transportation Research-D: Transport and environment

January 2000, vol. 5 nr. 2, pp. 121-135

1 This research was supported by the Environmental Report for Flanders (VMM), the Science Foundation of Flanders (FWO-project G .0063.98) and BOF fund of UFSIA.

2 The authors like to thank Ilse Bilsen and Ruth Creten for their contribution. 


\begin{abstract}
The assessment of uninterrupted traffic flow is traditionally based on empirical methods. We develop some analytic queueing models based on traffic counts and we model the behavior of traffic flows as a function of some of the most relevant determinants. These analytic models allow for parameterized experiments, which pave the way towards our research objectives: assessing what-if scenario's and sensitivity analysis for traffic management, congestion control, traffic design and the environmental impact of road traffic (e.g. emission models). The impact of some crucial modelling parameters is studied in detail and links with the broader research objectives are given. We illustrate our results for a highway, based on counted traffic flows in Flanders.
\end{abstract}

\title{
Keywords
}

Queueing theory, traffic flow modelling, congestion management 


\section{Introduction}

When modeling the environmental impact of road traffic, we can distinguish between both a static and dynamic impact of infrastructures and vehicles on emissions and waste (figure 1). On the one hand, roads can be considered as a visual intrusion. In addition, they may cause damage to natural watercourses or threaten the natural habitat of wildlife. Vehicles in turn consume natural resources and impose a strain on the environment at the end of their life cycle.

On the other hand, as they form part of traffic flows, infrastructures and vehicles also have a dynamic impact on the environment. Vehicles in use produce emissions and noise. Toxic fumes escape in the atmosphere when fuel tanks are filled, while driving leads to further emissions $\left(\mathrm{CO}_{2}, \mathrm{NO}_{\mathrm{x}}\right.$ and $\left.\mathrm{SO}_{2}\right)$ and dust (concrete, asphalt and rubber dust). Furthermore, an increase in garbage, accidents (physical and material damage) and, occasionally, distortion of infrastructures and nature elements (trees, animals,etc.) can be observed. Because traffic flows are a function of both the number of vehicles on the roads and the vehicle speed, the resulting environmental impact will also be a function of these parameters?

Figure 1: Static and dynamic impact of road traffic on emissions and waste

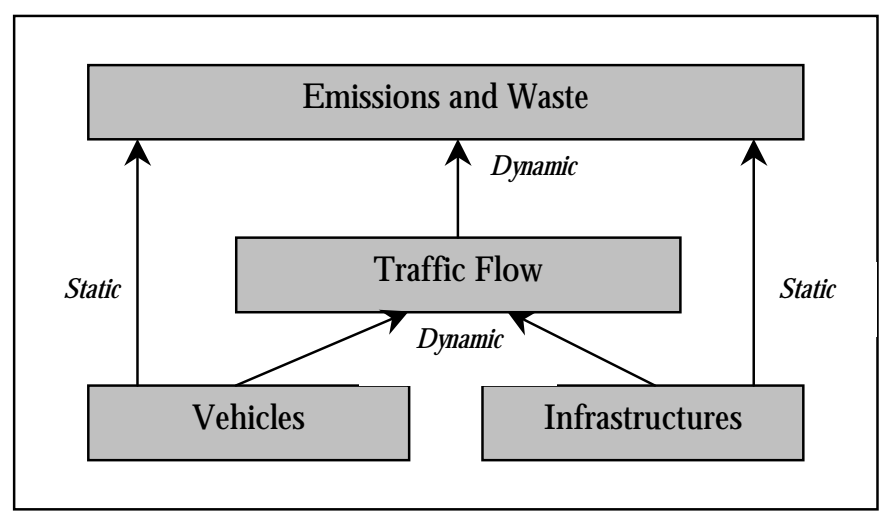

As traffic flows occupy a central position in the assessment of road traffic emissions, a robust traffic flow model is required. Traditionally, traffic flows are modeled empirically, using origindestination matrices. The objective of this approach is mainly explorative and explanatory. These descriptive models give an empirical justification of the well-known speed-flow and speed-density diagrams, but are limited in terms of predictive power and the possibility of sensitivity analysis. The construction of origin-destination matrices consists of five steps: transport generation, transport distribution, the modal split, assignment and calibration (Yang and Zhou,1998). An

\footnotetext{
3 In the long run, traffic flow, vehicles and infrastructures are interdependent. For example: due to the increased number of cars, government may decide to increase the number of lanes for particular highways, which leads to a change in traffic flows. Although it is possible to cope with these interactions, they are not considered in this article.
} 
alternative approach is to use speed-flow, speed-density and flow-density diagrams, in which data on traffic flows are collected and are fit into curves (Daganzo, 1997). The power of these two approaches lies in the description and explanation of traffic flows. Compared to these descriptive models, this paper presents a more operational approach using queueing theory.

Queueing theory is almost exclusively used to describe traffic behavior at signalized and unsignalized intersections (Heidemann, 1991, 1994, 1997). In this paper we will use queueing theory to describe uninterrupted traffic flows. The speed-flow-density diagrams will be constructed analytically, which makes it possible to assess the (future) environmental impact, perform sensitivity analyses and facilitate congestion management.

In the next section we will discuss several queueing models. The last section illustrates the two most realistic models with a real-life example.

\section{The Queueing Approach}

Queues occur whenever instantaneous demand exceeds the capacity to provide a service. Q ueueing theory involves the mathematical study of these waiting lines. Using a large number of alternative mathematical models, queueing theory provides various characteristics of the waiting line, like waiting time or length of the queue.

\subsection{Structure of a Queueing Model}

On of the most important equations in traffic flow theory incorporates the interdependence of traffic flow $q$, traffic density $\mathrm{E}$ and speed s:

$$
q=E * s
$$

When two of the three variables are known, the third variable can easily be obtained. If traffic count data are available, traffic flows can be assumed as given, which leaves us to calculate either traffic density or speed to complete the formula and use either as input for the appropriate queueing model. Table 1 gives an overview of the parameters used in this paper. 
Table 1: O verview of used parameters

\begin{tabular}{|l|l|}
\hline Parameter & D escription \\
\hline $\mathrm{E}$ & Traffic density $(\mathrm{veh} / \mathrm{km})$ \\
\hline $\mathrm{C}$ & Maximum traffic density $(\mathrm{veh} / \mathrm{km})$ \\
\hline $\mathrm{s}$ & Effective speed $(\mathrm{km} / \mathrm{h})$ \\
\hline $\mathrm{r}$ & Relative speed \\
\hline SN & Nominal speed $(\mathrm{km} / \mathrm{h})$ \\
\hline $\mathrm{q}$ & Traffic flow $(\mathrm{veh} / \mathrm{h})$ \\
\hline$\lambda$ & Arrival rate $(\mathrm{veh} / \mathrm{h})$ \\
\hline$\mu$ & Service rate $(\mathrm{veh} / \mathrm{h})$ \\
\hline$\rho$ & Traffic intensity $=\lambda / \mu$ \\
\hline $\mathrm{W}$ & Time in the system $(\mathrm{h})$ \\
\hline
\end{tabular}

In our model we define $C$ as the maximum traffic density 4 Roads are divided into segments of equal length $1 / C$, which matches the minimal length needed by one vehicle on that particular road. Each road segment is considered as a service station, in which vehicles arrive at rate $\lambda$ and get served at rate $\mu$ (figure 2).

Figure 2: Q ueueing representation of traffic flows

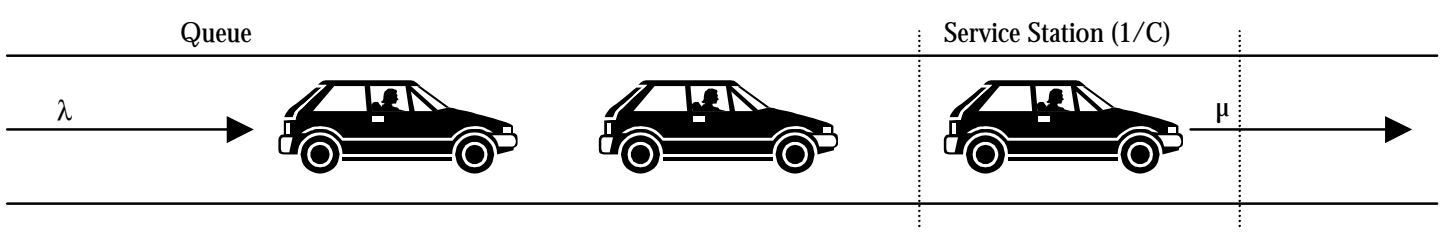

We define $\mathrm{W}$ as the total time a vehicle spends in the system, which equals the sum of waiting time (due to congestion) and service time. The higher the traffic intensity, the higher the time in the system becomes (the exact relation between $\mathrm{W}$ and $\rho$ depends upon the specific queueing model).

When $\mathrm{W}$ is known, the effective speed can easily be calculated as:

$$
s=\frac{1 / C}{W}
$$

The relative speed $r$, by definition, becomes:

$$
r=\frac{s}{S N}=\frac{1 / C}{W^{*} S N}
$$

Plotting the traffic flow, density and (relative and effective) speed on a graph gives us the wellknown speed-flow-density diagrams. The exact shape of these diagrams depends upon the queueing model and the characteristics of the arrival and service processes. In the sequel of this paper, we will develop the following queueing models (table 2) with their respective underlying

\footnotetext{
${ }^{4}$ The maximum traffic density depends on the type of road (number of lanes, etc.).
} 
assumptions. Queueing models are often refered to using the Kendall notation, consisting of several symbols - e.g. M/G/1. The first symbol is shorthand for the distribution of inter-arrival times, the second for the distribution of service times and the last one indicates the number of servers in the system.

Table 2: Characteristics of the used queueing models

\begin{tabular}{|l|l|l|}
\hline Model & Arrival Process & Service Process \\
\hline M/ M/1 & Poisson & Poisson \\
\hline M/ G/1 & Poisson & G eneral \\
\hline G/ G/ 1 & General & G eneral \\
\hline G/ G/ 1 State D ependent & General & G eneral \\
\hline
\end{tabular}

\subsection{The $M / M / 1$ Model}

The inter-arrival times are exponentially distributed (the arrival rate follows a Poisson distribution) with expected inter-arrival time equal to $1 / \lambda$ (with $\lambda$ equal to the product of the traffic density $\mathrm{E}$ and the nominal speed SN ). The service time delineates the time needed for a vehicle to pass one road segment and is exponentially distributed with expected service time $\mu$ (the service rate follows a Poisson distribution). When a vehicle drives at nominal speed SN , service time can be written as: $1 /\left(\mathrm{SN}^{*} \mathrm{C}\right)$ and $\mu$ equals the product of nominal speed SN with the maximum traffic density $C$.

Using these formulas for $\lambda$ and $\mu$, we obtain $\mathrm{W}$ as:

$$
W=\frac{1}{\mu-\lambda}=\frac{1}{S N *(C-E)}
$$

Using this expression for $\mathrm{W}$, the effective speed and relative speed are obtained:

$$
s=\frac{S N^{*}(C-E)}{C}=S N^{*}(1-\rho) \quad r=\frac{s}{S N}=1-\rho
$$

with $\rho$ the traffic intensity:

$$
\rho=\frac{\lambda}{\mu}=\frac{E}{C}
$$

Substituting for $E(=q / s)$ in (5) the following expression is obtained:

$$
f(s, q)=s^{2} * C-s^{*} C * S N+S N * q=0
$$

Maximizing $\mathrm{f}(\mathrm{s}, \mathrm{q})$ for $\mathrm{s}$ and substituting this value into (7), $\mathrm{q}_{\max }$ can be written as:

$$
q_{\max }=\frac{S N^{*} C}{4}
$$


From (5) we see that there exists a negative linear relationship between effective speed and traffic density E (figure 3).

Figure 3: The speed-density diagram for the M/ M/ 1 model

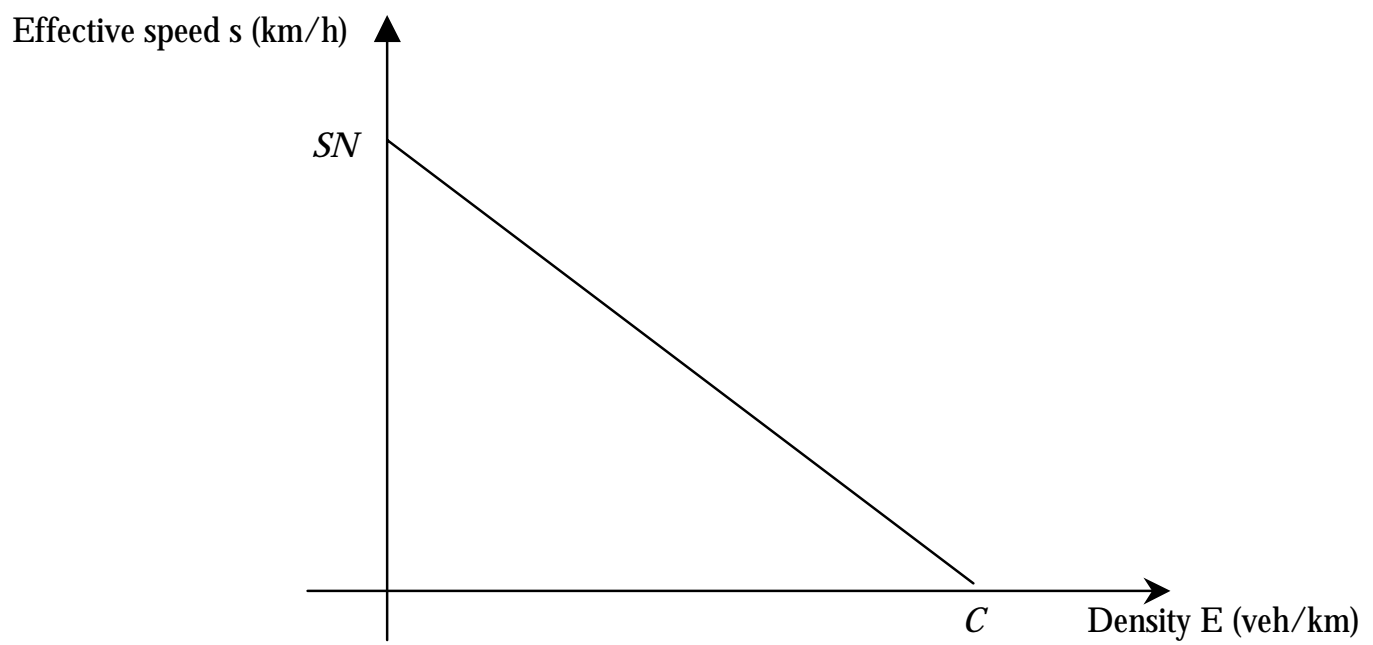

If traffic density is low, vehicles do not obstruct one another, which leads to higher effective speeds. When more vehicles arrive on the road, the effective speed s decreases. Using equation (1): $q=E * s$ and the above formula for $s$, we can construct the speed-flow (figure 4 ) and the flow-density diagrams (figure 5) for the M/ M/ 1 model.

Figure 4: The speed-flow diagram for the M/ M/ 1 model

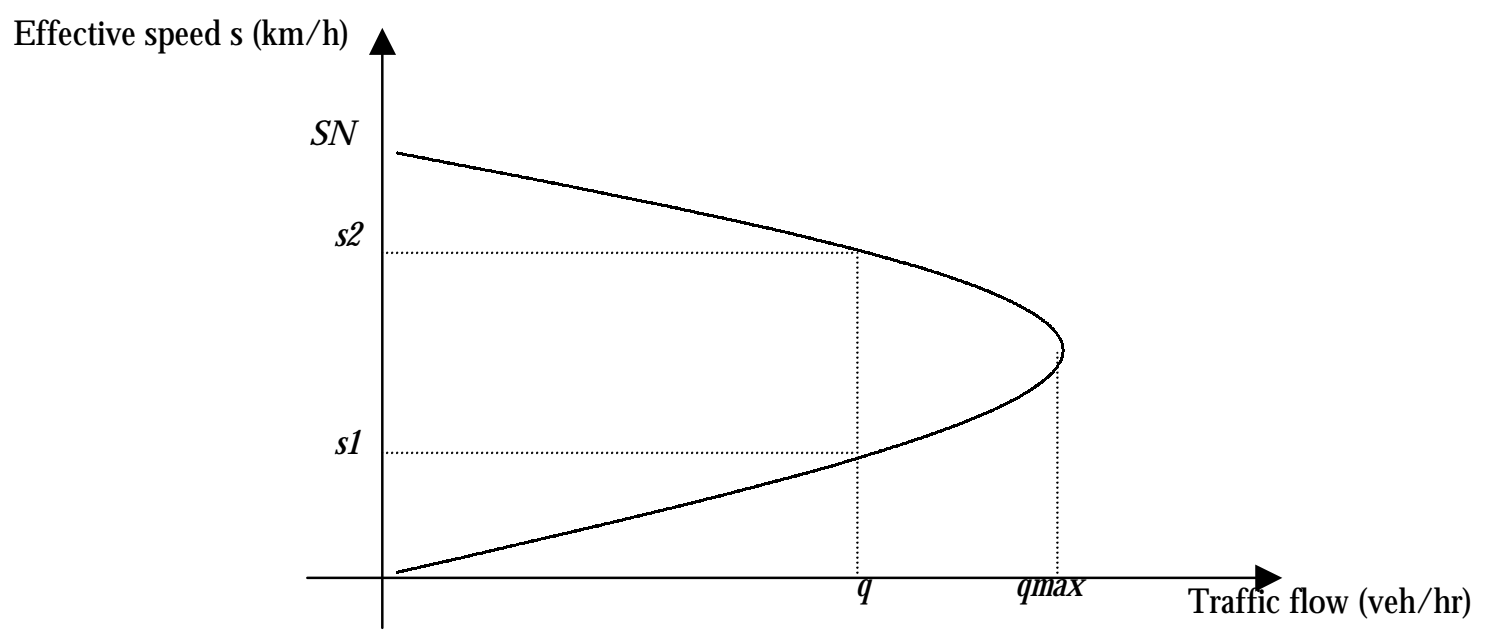

The speed-flow diagram is the envelope of all possible combinations of the effective speed and traffic flow. Figure 4 also illustrates that although every speed s corresponds with one unique traffic flow q, the reverse is not true. There are two speeds for every traffic flow: an upper branch (s2) where speed decreases with flow and an lower branch (s1) with an increasing speed in terms of flow. An intuitive explanation can be as follows: as the flow moves from SN to $q_{\max }$, congestion increases but the flow rises because the decline in speed is offset by the higher 
volume. If traffic continues to enter the flow past $q_{\max }$, flow falls because the decline in speed more than offsets the additional vehicle numbers further increasing congestion (D aganzo, 1997). An equivalent representation is the flow-density diagram (figure 5). This diagram can be interpreted in the same way as the speed-flow diagram: the left side of the graph (E 1) corresponds to the upper part of figure 4, the right side (E 2) to the lower part.

Figure 5: The flow-density diagram for the M/ M/ 1 model

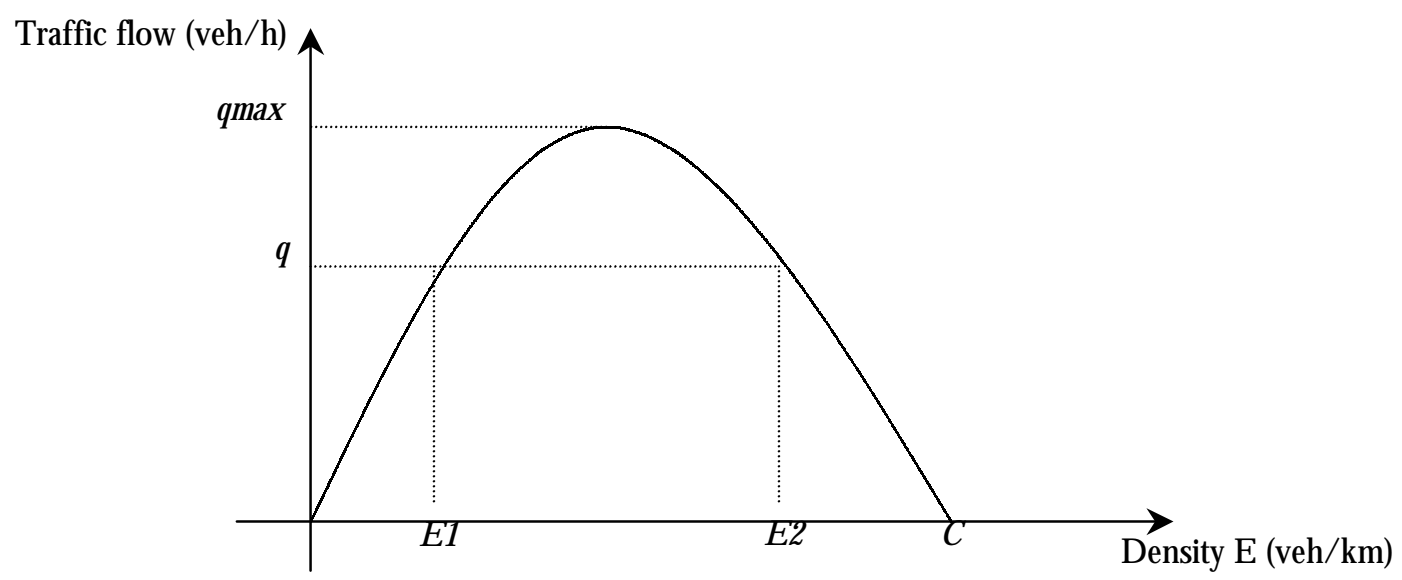

The M/ M/ 1 model is interesting as a base case, but is inadequate to represent real-life traffic flows. In the next two sections we will relax the M/ M/ 1 model: first, the service times follow a general distribution (M/G/1) and, secondly, both arrival and service times follow a general distribution $(G / G / 1)$.

\subsection{The M/G/ 1 Model}

As in the M/M/ 1 model inter-arrival times follow an exponential distribution with expected inter-arrival time $1 / \lambda, \lambda$ being the product of traffic density and nominal speed. The service time however is generally distributed with an expected service time of $1 / \mu$ and a standard deviation of $\sigma$. Expected service rate is $\mu$, which equals the product of nominal speed SN with maximum traffic density $C$.

Combining Little's theorem and the Pollaczek-Khintchine formula for $\mathrm{L}^{\mathrm{B}}$ (Hillier and Liebermann, 1995) and substituting for $\lambda$ and $\mu$, we obtain the following formula for the total time in the system $\mathrm{W}$ :

$$
W=\frac{1}{S N * C}+\frac{\rho^{2}+S N^{2} * E^{2} * \sigma^{2}}{2 * S N^{*} E^{*}(1-\rho)}
$$

${ }^{5} \mathrm{~L}$ defined as the average number of cars in the system. 
Using the above expression for $\mathrm{W}$, effective and relative speed can be calculated in an analog way as in the M/ M/ 1 model:

$$
s=\frac{2 * S N *(C-E)}{2 * C+E *\left(\beta^{2}-1\right)}=\frac{2 * S N^{*}(1-\rho)}{2+\rho *\left(\beta^{2}-1\right)} \quad r=\frac{2 *(1-\rho)}{2+\rho^{*}\left(\beta^{2}-1\right)}
$$

with $\beta$ delineating the coefficient of variation of service time (or $\beta=\sigma^{*} \mathrm{SN} * \mathrm{C}$ ).

Using these formulas we can construct the speed-flow, speed-density and flow-density diagrams for the M/G/ 1 model. The exact shape of these curves depends upon the variation coefficient of the service time, $\beta$ (figure 6).

Figure 6: The speed-density diagram for the M/ G/ 1 model with $\beta<1, \beta=1$ and $\beta>1$

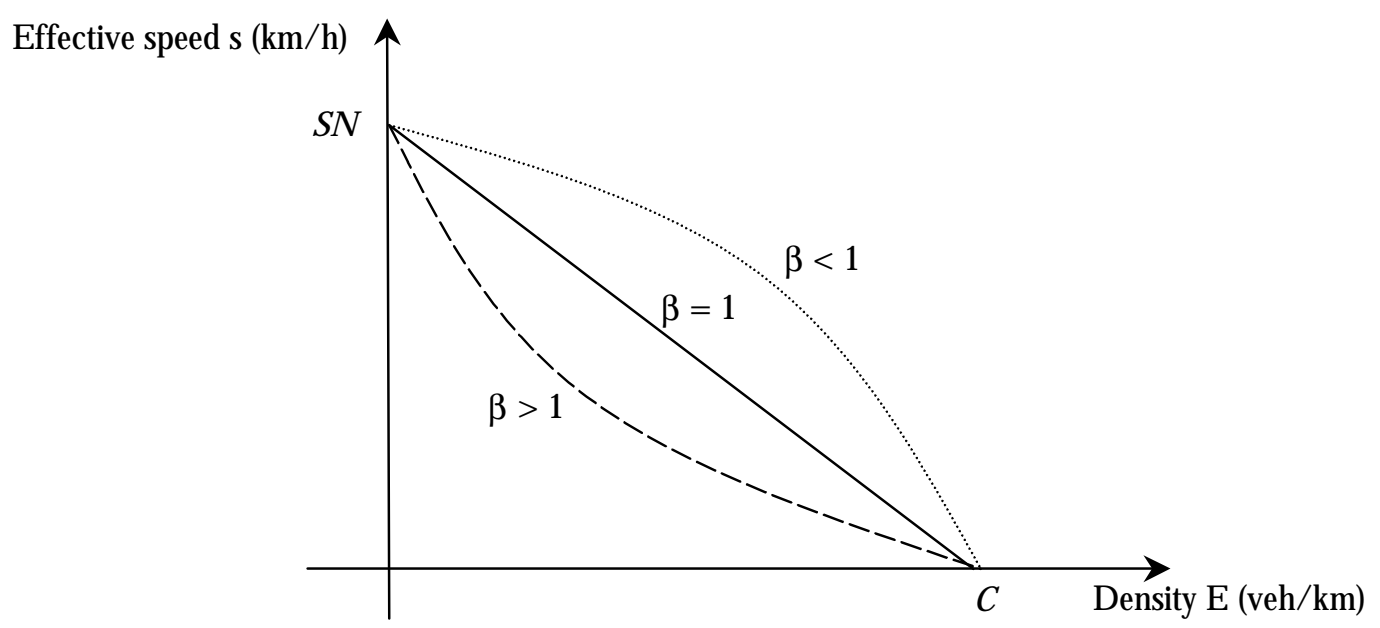

Substituting E (= q/ s) in above formula (8) and rewriting, the following expression for the speedflow diagram is obtained:

$$
f(s, q)=2 * C * s^{2}+\left[q *\left(\beta^{2}-1\right)-2 * C * S N\right] * s+2 * q * S N=0
$$

Maximizing this equation for s, we can calculate the maximum traffic flow $\left(\mathrm{q}_{\max }\right)$ :

$$
\begin{array}{ll}
q_{\max }=2 * S N^{*} C *\left[\frac{\sqrt{\beta^{2}+1}-\sqrt{2}}{\beta-1}\right]^{2} & \beta \geq 0 \\
q_{\max }=\frac{S N^{*} C}{4} & \beta=1
\end{array}
$$

The value of $\mathrm{q}_{\max }$ is a function of the variation parameter $\beta$. 


\subsection{The G/G/ 1 Model}

With the G/G/ 1 model both arrival times and service times follow a general distribution with expected arrival time $1 / \lambda$ and standard deviation $\sigma_{a}$, expected service times $1 / \mu$ and standard deviation of $\sigma_{\mathrm{b}}$ respectively. Consequently, the shape of the speed-flow-density diagrams will depend not only on the variance of the service times but also on the variance of the inter-arrival times.

Combining Little's theorem and the Kraemer-Lagenbach-Belz (Kraemer and Lagenbach-Belz, 1976) formula for $L$ and substituting for $\lambda$ and $\mu$, we obtain the following formulas for the total time in the system $\mathrm{W}$ :

$$
\begin{aligned}
& W=\frac{1}{S N^{*} C}+\frac{\rho^{2} *\left(c_{a}^{2}+c_{s}^{2}\right)}{2 * S N^{*} E^{*}(1-\rho)} * e^{\frac{-2^{*}(1-\rho)^{*}\left(1-c_{a}^{2}\right)^{2}}{3^{*} \rho^{*}\left(c_{a}^{2}+c_{s}^{2}\right)}}, c_{a}^{2} \leq 1 \\
& W=\frac{1}{S N^{*} C}+\frac{\rho^{2} *\left(c_{a}^{2}+c_{s}^{2}\right)}{2 * S N^{*} E^{*}(1-\rho)} * e^{\frac{-(1-\rho)^{*}\left(c_{a}^{2}-1\right)^{2}}{(1+\rho)^{*}\left(c_{a}^{2}+10 c_{s}^{2}\right)}}, c_{a}^{2}>1
\end{aligned}
$$

with $c_{a}^{2}$ representing the squared coefficient of variation of inter-arrival times and $c_{b}^{2}$ the squared coefficient of variation of service time.

Using (5) and the above expressions for $\mathrm{W}$, the effective speed formulas become:

$$
\begin{aligned}
& s=\frac{2 * S N^{*}(1-\rho)}{2 *(1-\rho)+\rho *\left(c_{a}^{2}+c_{s}^{2}\right) * e^{\frac{-2^{*}(1-\rho)^{*}\left(1-c_{a}^{2}\right)^{2}}{3^{*}\left(c_{a}^{2}+c_{s}^{2}\right)}}} \quad c_{a}^{2} \leq 1 \\
& s=\frac{2 * S N *(1-\rho)}{2 *(1-\rho)+\rho *\left(c_{a}^{2}+c_{s}^{2}\right) * e^{\frac{-(1-\rho)^{*}\left(c_{a}^{2}-1\right)^{2}}{\left(1+c_{a}^{2}+10 c_{s}^{2}\right)}}} \quad c_{a}^{2}>1
\end{aligned}
$$

The exact shape of the diagrams depends not only on the variation coefficient of service times but also on the variation coefficient of inter-arrival times. In practice, these formulas generate no explicit solution and are numerically obtained by the Newton-Raphson method (Wagner, 1975). See appendix for an overview of the Newton - Raphson method.

\subsection{State Dependent G/ G/ 1 Model}

Instead of using a fixed service rate $\mu$, we now make the service rate a function of the traffic flow q. Vehicles are served at a certain rate dependent upon the number of vehicles in the system. We established that $\mu=\mathrm{C} * \mathrm{SN}$. In the state dependent G/G/1 model we assume that the 
nominal speed $\mathrm{SN}$ is a function of the traffic flow or $\mathrm{SN}=\mathrm{f}(\mathrm{q})$. This function can either be linear or exponential (Jain and Smith, 1997).

The linear function is written as follows (Jain and Smith, 1997):

$$
S N_{q}=S N *\left[\frac{q_{\max }+1-q}{q_{\max }}\right]
$$

The exponential form we use is the following:

$$
S N_{q}=S N * e^{-a^{*}\left(\frac{q}{q_{\max }}\right)}
$$

with $\mathrm{SN}_{\mathrm{q}}$ delineating the nominal speed that occurs with $\mathrm{q}$ vehicles in the system and a the pressure coefficient for the exponential function.

Figure 7 shows a graphical representation of both functions. The shape of the exponential function will depend upon the pressure coefficient a. When this coefficient increases, the faster the nominal speed declines at an increased rate.

Figure 7: Linear and different exponential functions for the nominal speed $\mathrm{SN}_{\mathrm{q}}$

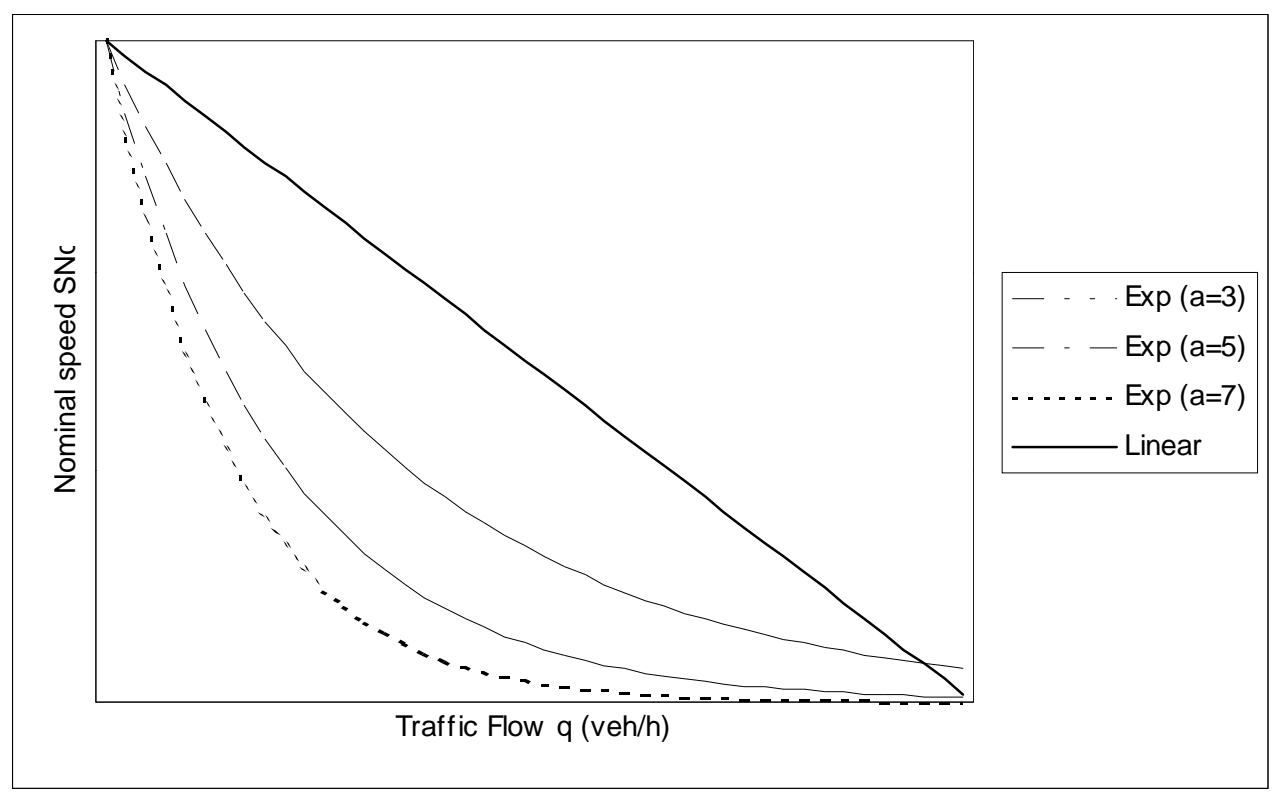

Plugging these functions in our G / G/ 1 model leads to the state dependent model. 


\section{Applications}

This application shows some results of the G/G/1 model and the state dependent G/G/1 model for highway E 19, from St-Job to Merksem (Antwerp).

In this example we make the nominal speed SN equal to the maximum speed allowed on a highway in Belgium (or $120 \mathrm{~km} / \mathrm{h}$ ). We dispose of hourly traffic counts for each weekday for the years 1993, 1994 and 1995. Figure 7 shows the average traffic flow over these three years per hour. Traffic flow peaks at 8.00 a.m. with 3200 vehicles per hour and then remains fairly constant at approximately 2000 vehicles per hour until 7.00 p.m..

Figure 8: Traffic flow (veh/h) on highway E19

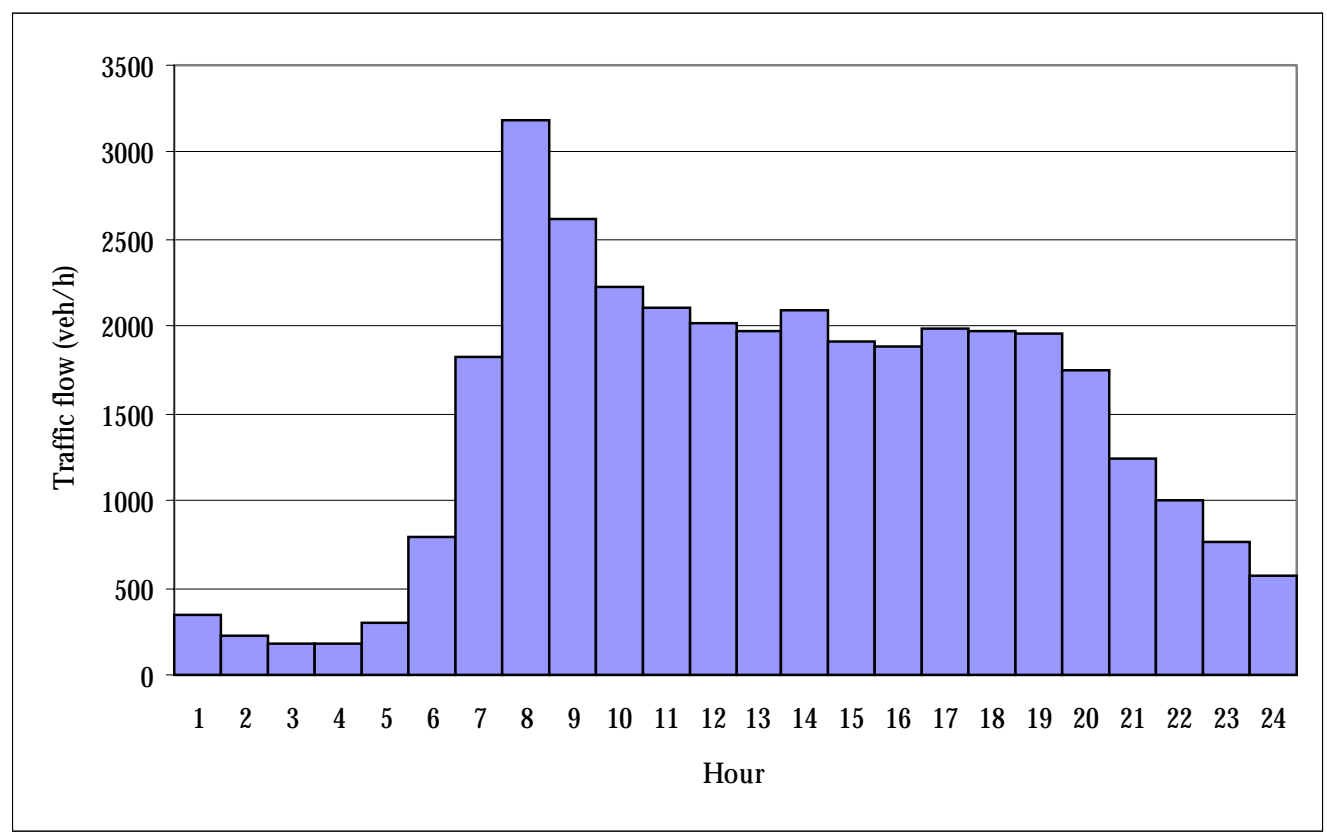

The maximum traffic flow observed in the three years considered is 4350 vehicles per hour. Assuming that this flow occurred in the best possible conditions (e.g. good weather), we calculate the maximum traffic density $C$ with $\mathrm{q}_{\max }$ equal to 4350 and the variation coefficients both equal to 0.5 (low variance). $\mathrm{C}$ is then equal to 74 vehicles per kilometer.

\subsection{G/ G/ 1 Model}

Using formulas (14) we can construct the speed-flow-density diagrams for different values of the variation parameters. Figure 9 shows the speed-flow diagram: lower variance corresponds with higher speeds and more cars being able to flow through the system. 
Figure 9: The speed-flow diagram for highway E19

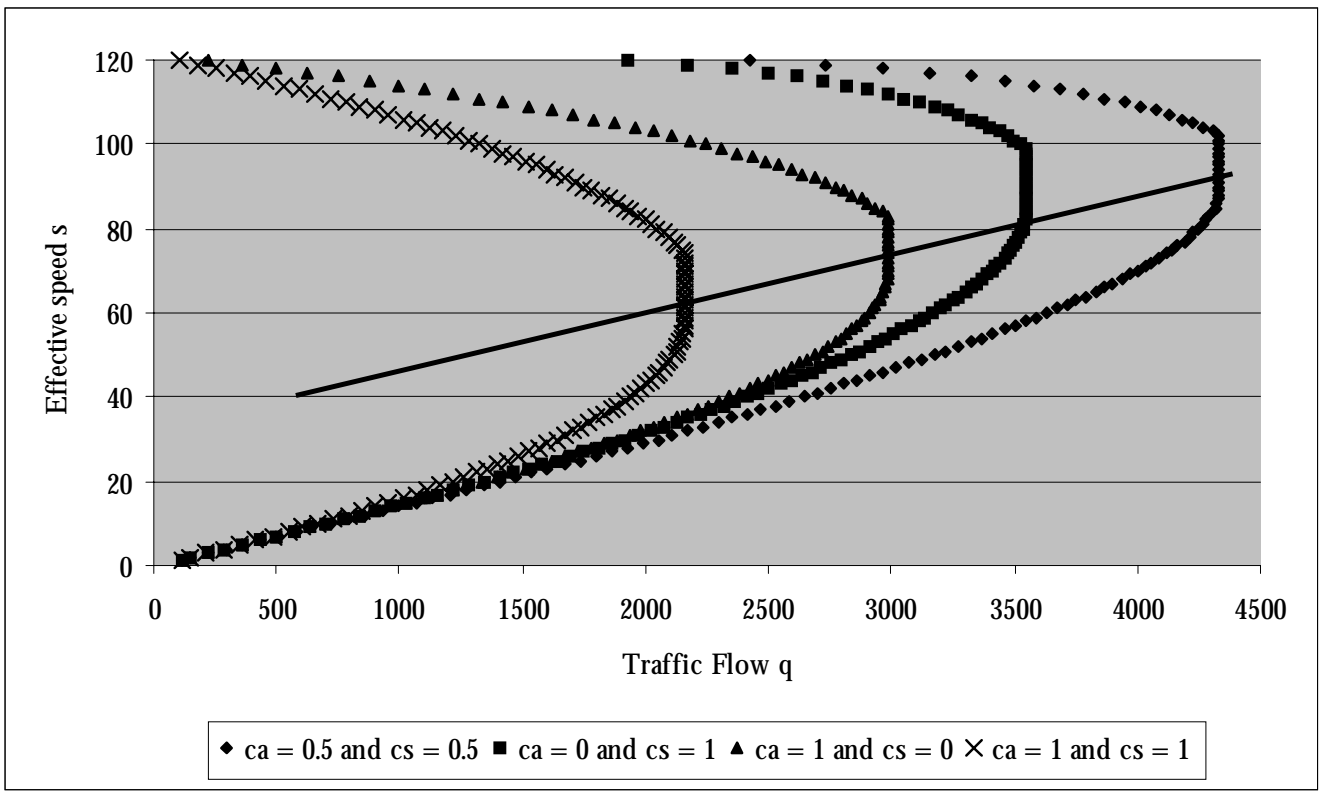

We see that the variance on the arrival rate $\left(c_{A}=1\right.$ and $\left.c_{s}=0\right)$ has a larger impact than the variance on the service rate $\left(c_{A}=0\right.$ and $\left.c_{s}=1\right)$. Actions to increase traffic flow should primarily be focused on the arrival rate variance. A similar conclusion can be obtained using the flowdensity diagram (figure 10).

Figure 10: The flow-density diagram for highway E19

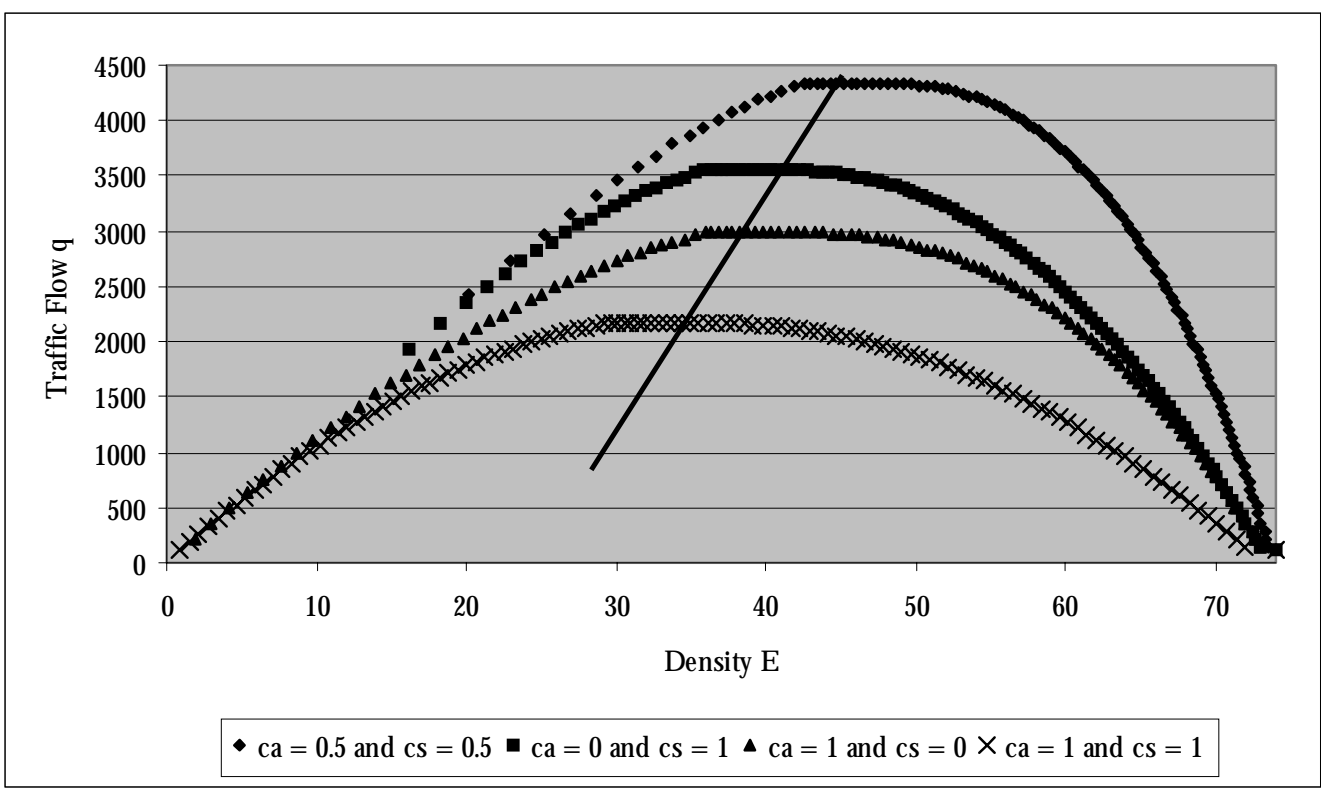


Finally, the speed-density diagram is constructed for a given density of 40 vehicles per km: we see that the effective speed ranges from approximately 50 (high variance) to approximately 110 (low variance) $\mathrm{km} / \mathrm{h}$.

Figure 11: the speed-density diagram for highway E 19

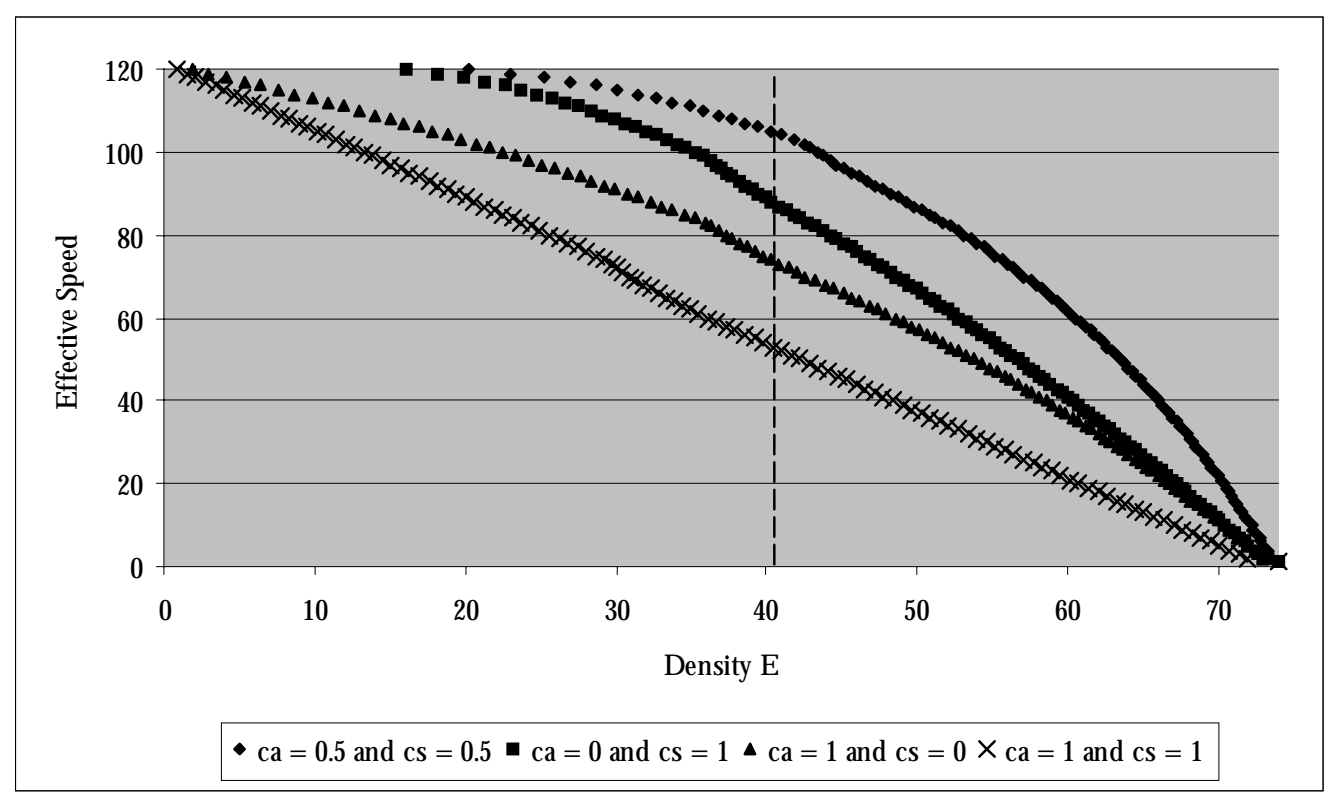

Using the above constructed diagrams and the traffic flow data for this highway (figure 8), we can calculate for each traffic flow $q$ the speeds $s_{1}$ and $s_{2}$. Table 3 shows the results obtained from the G/ G/ 1 model, using the different variation parameters $c_{a}$ and $c_{s}$. 
Table 3: Upper and lower speeds for highway E19, using different variation parameters

\begin{tabular}{|c|c|c|c|c|c|c|c|c|c|c|c|c|c|}
\hline \multirow{2}{*}{\multicolumn{2}{|c|}{ qmax }} & \multicolumn{3}{|l|}{4350} & & \multirow{2}{*}{\multicolumn{3}{|c|}{2983}} & \\
\hline & & \multicolumn{3}{|l|}{$\mathrm{ca}=0,5$ en $\mathrm{cs}=0,5$} & \multicolumn{3}{|l|}{$\mathrm{ca}=0$ en $\mathrm{CS}=1$} & & & & \multicolumn{3}{|l|}{$\mathrm{ca}=1$ en $\mathrm{CS}=1$} \\
\hline Hour & $\mathrm{q}(\mathrm{veh} / \mathrm{h})$ & q/ qmax & s1 & $\mathrm{s2}$ & q/ qmax & s1 & $\mathrm{s} 2$ & q/ qmax & s1 & s2 & q/ qmax & s1 & s2 \\
\hline 1 & 340 & $8 \%$ & 5 & 120 & $10 \%$ & 5 & 120 & $11 \%$ & 5 & 119 & $16 \%$ & 5 & 117 \\
\hline 2 & 225 & $5 \%$ & 3 & 120 & $6 \%$ & 3 & 120 & $8 \%$ & 3 & 120 & $10 \%$ & 3 & 118 \\
\hline 3 & 178 & $4 \%$ & 2 & 120 & $5 \%$ & 2 & 120 & $6 \%$ & 2 & 120 & $8 \%$ & 2 & 119 \\
\hline 4 & 180 & $4 \%$ & 2 & 120 & $5 \%$ & 2 & 120 & $6 \%$ & 2 & 120 & $8 \%$ & 3 & 119 \\
\hline 5 & 303 & $7 \%$ & 4 & 120 & $9 \%$ & 4 & 120 & $10 \%$ & 4 & 119 & $14 \%$ & 4 & 117 \\
\hline 6 & 787 & $18 \%$ & 11 & 120 & $22 \%$ & 11 & 120 & $26 \%$ & 11 & 116 & $36 \%$ & 12 & 110 \\
\hline 7 & 1826 & $42 \%$ & 26 & 120 & $51 \%$ & 28 & 120 & $61 \%$ & 29 & 106 & $84 \%$ & 36 & 88 \\
\hline 8 & 3180 & $73 \%$ & 50 & 117 & $90 \%$ & 60 & 109 & $107 \%$ & $*$ & * & $147 \%$ & * & $*$ \\
\hline 9 & 2612 & $60 \%$ & 39 & 119 & $74 \%$ & 45 & 116 & $88 \%$ & 47 & 95 & $121 \%$ & $*$ & $*$ \\
\hline 10 & 2235 & $52 \%$ & 33 & 120 & $63 \%$ & 36 & 119 & $75 \%$ & 37 & 100 & $103 \%$ & $*$ & $*$ \\
\hline 11 & 2109 & $49 \%$ & 31 & 120 & $59 \%$ & 34 & 119 & $71 \%$ & 35 & 102 & $97 \%$ & 50 & 77 \\
\hline 12 & 2016 & $47 \%$ & 29 & 120 & $57 \%$ & 32 & 120 & $68 \%$ & 33 & 103 & $93 \%$ & 44 & 81 \\
\hline 13 & 1978 & $46 \%$ & 29 & 120 & $56 \%$ & 31 & 120 & $66 \%$ & 32 & 104 & $91 \%$ & 42 & 83 \\
\hline 14 & 2095 & $48 \%$ & 31 & 120 & $59 \%$ & 33 & 119 & $70 \%$ & 34 & 102 & $97 \%$ & 49 & 78 \\
\hline 15 & 1911 & $44 \%$ & 28 & 120 & $54 \%$ & 30 & 120 & $64 \%$ & 31 & 105 & $88 \%$ & 39 & 85 \\
\hline 16 & 1892 & $44 \%$ & 27 & 120 & $53 \%$ & 30 & 120 & $63 \%$ & 30 & 105 & $87 \%$ & 38 & 86 \\
\hline 17 & 1987 & $46 \%$ & 29 & 120 & $56 \%$ & 31 & 120 & $67 \%$ & 32 & 104 & $92 \%$ & 42 & 82 \\
\hline 18 & 1977 & $46 \%$ & 29 & 120 & $56 \%$ & 31 & 120 & $66 \%$ & 32 & 104 & $91 \%$ & 42 & 83 \\
\hline 19 & 1964 & $45 \%$ & 29 & 120 & $55 \%$ & 31 & 120 & $66 \%$ & 32 & 104 & $91 \%$ & 41 & 83 \\
\hline 20 & 1755 & $40 \%$ & 25 & 120 & $49 \%$ & 27 & 120 & $59 \%$ & 27 & 106 & $81 \%$ & 33 & 90 \\
\hline 21 & 1243 & $29 \%$ & 18 & 120 & $35 \%$ & 18 & 120 & $42 \%$ & 18 & 112 & $57 \%$ & 21 & 102 \\
\hline 22 & 1008 & $23 \%$ & 14 & 120 & $28 \%$ & 15 & 120 & $34 \%$ & 15 & 114 & $47 \%$ & 16 & 106 \\
\hline 23 & 762 & $18 \%$ & 11 & 120 & $21 \%$ & 11 & 120 & $26 \%$ & 11 & 116 & $35 \%$ & 12 & 110 \\
\hline 24 & 562 & $13 \%$ & 8 & 120 & $16 \%$ & 8 & 120 & $19 \%$ & 8 & 117 & $26 \%$ & 8 & 113 \\
\hline
\end{tabular}

The results can easily be compared with the constructed speed-flow-density diagrams. For the case with high variances ( $c_{a}$ and $c_{s}$ both equal to one), at hour 8.00, 9.00 and 10.00 a.m., the observed traffic flow becomes larger than the maximum possible traffic flow on the highway given these variance parameters. Consequently there are no speeds that can be calculated for these instances.

\subsection{State Dependent G/ G/ 1 Model}

Using the preceding linear and exponential functions, we now illustrate the state dependent G/G/ 1 model with the same example.

The speed-density diagram shown in figure 12 shows that speed lowers fast in the beginning and then remains at a constant level of approximately $20 \mathrm{~km} / \mathrm{h}$ if we employ the exponential model. The linear model, by contrast, shows a more gradually decreasing speed. 
Figure 12: The speed-density diagram for the State dependent G/ G / 1 model

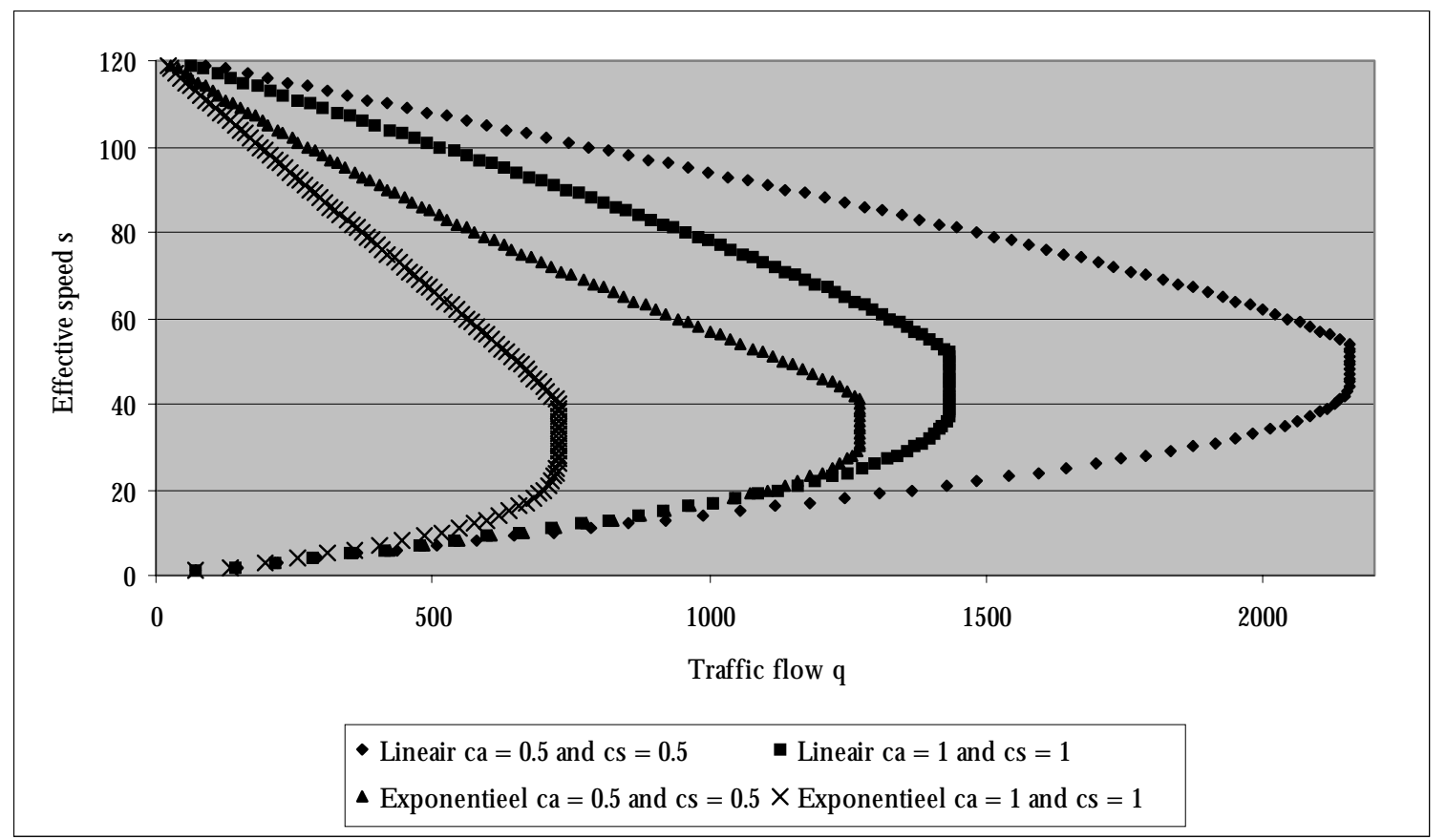

The speed-flow and flow-density diagrams can be constructed in the same way. Using the diagrams and the traffic flow data for this highway (figure 8), we can calculate for each traffic flow q the speeds $s_{1}$ and $s_{2}$ for this model. Table 4 shows the results obtained from the state dependent G/G/ 1 model, using the different variation parameters $c_{a}$ and $c_{s}$ and the pressure coefficient of 5 (see figure 7). 
Table 4: Upper and lower speeds for highway E19, using different variation parameters

\begin{tabular}{|c|c|c|c|c|c|c|c|c|c|c|c|c|c|}
\hline & qmax & 2397 & & & 1418 & & & 2805 & & & 1494 & & \\
\hline & & $\begin{array}{l}\text { Linear } c a=0,5 \text { en } \\
\mathrm{cs}=0,5\end{array}$ & & & $\mathrm{ca}=1 \mathrm{en}$ & & & $\mathrm{ca}=0,5$ & & & ial ca $=1$ & & \\
\hline Hour & $\mathrm{eh} / \mathrm{h})$ & q/ qmax & s1 & & q/ qmax & s1 & s2 & q/ qmax & s1 & s2 & q/ qmax & s1 & $\mathrm{s} 2$ \\
\hline 1 & 409 & $17 \%$ & 5 & 112 & $29 \%$ & 5 & 107 & $15 \%$ & 5 & 95 & $27 \%$ & 5 & 94 \\
\hline 2 & 294 & $12 \%$ & 3 & 115 & $21 \%$ & 3 & 112 & $10 \%$ & 3 & 103 & $20 \%$ & 3 & 103 \\
\hline 3 & 232 & $10 \%$ & 2 & 117 & $16 \%$ & 2 & 114 & $8 \%$ & 2 & 107 & $16 \%$ & 3 & 106 \\
\hline 4 & 214 & $9 \%$ & 2 & 117 & $15 \%$ & 3 & 114 & $8 \%$ & 2 & 107 & $14 \%$ & 3 & 106 \\
\hline 5 & 297 & $12 \%$ & 4 & 113 & $21 \%$ & 4 & 109 & $11 \%$ & 4 & 98 & $20 \%$ & 4 & 97 \\
\hline 6 & 657 & $27 \%$ & 11 & 100 & $46 \%$ & 12 & 88 & $23 \%$ & 12 & 68 & $44 \%$ & 14 & 65 \\
\hline 7 & 1393 & $58 \%$ & 29 & 69 & $98 \%$ & * & * & $50 \%$ & * & * & $93 \%$ & $*$ & * \\
\hline 8 & 2360 & $98 \%$ & $*$ & $*$ & $166 \%$ & $*$ & $*$ & $84 \%$ & $*$ & $*$ & $158 \%$ & $*$ & * \\
\hline 9 & 2107 & $88 \%$ & $*$ & $*$ & $149 \%$ & $*$ & $*$ & $75 \%$ & $*$ & $*$ & $141 \%$ & * & * \\
\hline 10 & 2015 & $84 \%$ & $*$ & $*$ & $142 \%$ & $*$ & $*$ & $72 \%$ & $*$ & $*$ & $135 \%$ & * & * \\
\hline 11 & 1991 & $83 \%$ & 39 & 57 & $140 \%$ & $*$ & $*$ & $71 \%$ & $*$ & $*$ & $133 \%$ & * & * \\
\hline 12 & 1931 & $81 \%$ & 34 & 61 & $136 \%$ & $*$ & $*$ & $69 \%$ & $*$ & $*$ & $129 \%$ & * & * \\
\hline 13 & 1880 & $78 \%$ & 33 & 63 & $133 \%$ & * & $*$ & $67 \%$ & $*$ & * & $126 \%$ & * & * \\
\hline 14 & 1988 & $83 \%$ & 38 & 57 & $140 \%$ & $*$ & $*$ & $71 \%$ & * & * & $133 \%$ & * & * \\
\hline 15 & 1796 & $75 \%$ & 31 & 65 & $127 \%$ & * & $*$ & $64 \%$ & * & * & $120 \%$ & * & * \\
\hline 16 & 1723 & $72 \%$ & 30 & 66 & $121 \%$ & $*$ & $*$ & $61 \%$ & * & $*$ & $115 \%$ & $*$ & * \\
\hline 17 & 1806 & $75 \%$ & 33 & 62 & $127 \%$ & $*$ & $*$ & $64 \%$ & * & $*$ & $121 \%$ & $*$ & * \\
\hline 18 & 1856 & $77 \%$ & 33 & 63 & $131 \%$ & $*$ & $*$ & $66 \%$ & * & $*$ & $124 \%$ & $*$ & * \\
\hline 19 & 1848 & $77 \%$ & 33 & 63 & $130 \%$ & $*$ & * & $66 \%$ & * & $*$ & $124 \%$ & $*$ & * \\
\hline 20 & 1676 & $70 \%$ & 27 & 71 & $118 \%$ & * & * & $60 \%$ & $*$ & * & $112 \%$ & * & * \\
\hline 21 & 1223 & $51 \%$ & 18 & 87 & $86 \%$ & 24 & 65 & $44 \%$ & 27 & 43 & $82 \%$ & $*$ & * \\
\hline 22 & 1022 & $43 \%$ & 14 & 94 & $72 \%$ & 17 & 77 & $36 \%$ & 17 & 57 & $68 \%$ & 22 & 51 \\
\hline 23 & 777 & $32 \%$ & 11 & 100 & $55 \%$ & 12 & 89 & $28 \%$ & 12 & 69 & $52 \%$ & 13 & 66 \\
\hline 24 & 572 & $24 \%$ & 8 & 106 & $40 \%$ & 8 & 98 & $20 \%$ & 8 & 81 & $38 \%$ & 9 & 79 \\
\hline
\end{tabular}

As in the previous example we see that there are many speeds that cannot be calculated as a consequence of the number of vehicles being larger than the maximum vehicles $q_{\max }$. This could happen for instance in case of a snowstorm.

\section{Conclusions}

Based on queueing theory we analytically constructed the well-known speed-flow-density diagrams. Using several queueing models, speed is determined, based on different arrival and service processes. The exact shape of the different speed-flow-density diagrams is largely determined by the model parameters. Therefore we believe that a good choice of parameters can help to adequately describe reality. We illustrated this with an example, using the most general models (including a state dependent model) for a highway. Due to the fact that speeds have a 
significant influence on vehicle emissions, our models can be effectively used to assess the environmental impact of road traffic.

The authors like to thank two anonymous referees for their valuable comments.

\section{Appendix}

This appendix is based on Wagner (1975).

Nonlinear functions $d s$ ) which have no anlytical solution, are often solved using the following standardized format:

Step 1: Select an arbitrary initial trial point $s^{0}$;

Step 2: Terminate the iterations if $\frac{\partial c}{\partial s_{j}}=0$, for $\mathrm{j}=1,2, \ldots, \mathrm{n}$ at the trial point $\mathrm{s}^{\mathrm{k}}$. Otherwise, determine values $y_{j}^{k}$, for $\mathrm{j}=1,2, \ldots, \mathrm{n}$ and continue to step 3;

Step 3: Calculate a new trial point: $s_{j}^{k+1}=s_{j}^{k}+y_{j}^{k}$, for $\mathrm{j}=1,2, \ldots, \mathrm{n}$.

Various algorithms for selecting $y_{j}^{k}$, are available. Many of these methods consist in the combination of the direction $d_{j}^{k}$, and the step size $t^{k}$. Direction is based on information about how $d s$ ) behaves near the trial point $s^{k}$, thus based on the local properties of $\left.d s\right)$. Step size depends on how $d(s)$ changes when proceeding in the selected direction $d_{j}^{k}$. Then, $y_{j}^{k}$ is calculated as: $y_{j}^{k}=t^{k} * d_{j}^{k}$.

The Newton - Rapson method calculates the direction $d_{j}^{k}$ as a quadratic fit of the objective function. This method results in using the second-order partial derivatives to form a weighted average of the first-order partial derivatives. Specifically, the $d_{j}^{k}$ are found by solving following linear system: $\sum \frac{\partial^{2} c}{\partial s_{i} \partial s_{j}} d_{j}=-\frac{\partial c}{\partial s_{i}}$. 


\section{References}

Daganzo, C.F., 1997, Fundamentals of Transportation and Traffic Operations, Elsevier Science Ltd., Oxford.

Heidemann, D., 1991, Queue length and waiting-time distributions at priority intersections, Transportation Research-B, 25, 163-174.

Heidemann, D., 1994, Queue length and delay distributions at traffic signals, Transportation Research-B, 28B, 377-389.

Heidemann, D., 1996, A queueing theory approach to speed-flow-density relationships, Transportation and Traffic Theory, Proceedings of the 13th International Symposium on Transportation and Traffic Theory, Lyon, France, 14-26 July 1996.

Heidemann, D . and Wegmann H., 1997, Queueing at unsignalized intersections, Transportation Research - B, 31, 239-263.

Hillier F.S and Liebermann G.J., 1995, Introduction to O perations Research, McG raw-Hill, New York.

Jain, R. and Smith J.M., 1997, Modelling vehicular traffic flow using M/ G / C/ C state dependent queueing models, Transportation Science, 31, 324-336.

Kraemer and Lagenbach-Belz, 1976, Approximate formulae for the delay in the queueing system G I/ GI/ 1, Conference book, p. 235-1/ 8, Melbourne, Eight International Teletraffic Congress.

Van Zuylen H.J. and Branston D.M., 1982, Consistent link flow estimation from counts, Transportation Research-B, 16B, 473-476.

Wagner H.M., 1975, Principles of O perations Research, New Jersey.

Willumsen, L.G., 1981, Simplified transport models based on traffic counts, Transportation, 10, 257-278.

Yang H. and Zhou J., 1998, Optimal traffic counting locations for origin-destination matrix estimation, Transportation Research-B, 32, 109-126 